\title{
MyoSpring: 3D Printing Mechanomyographic Sensors for Subtle Finger Gesture Recognition
}

\author{
Stephen Shiao-ru Lin \\ School of Computer Science, University of Sydney \\ Sydney, Australia \\ stephen.lin@sydney.edu.au \\ Kithmini Herath* \\ School of Computer Science, University of Sydney \\ Sydney, Australia \\ kithmini.herath@sydney.edu.au
}

\author{
Nisal Menuka Gamage \\ School of Computer Science, University of Sydney \\ Sydney, Australia \\ nisal.gamage@sydney.edu.au \\ Anusha Withana ${ }^{\dagger}$ \\ School of Computer Science, University of Sydney \\ Sydney, Australia \\ anusha.withana@sydney.edu.au
}
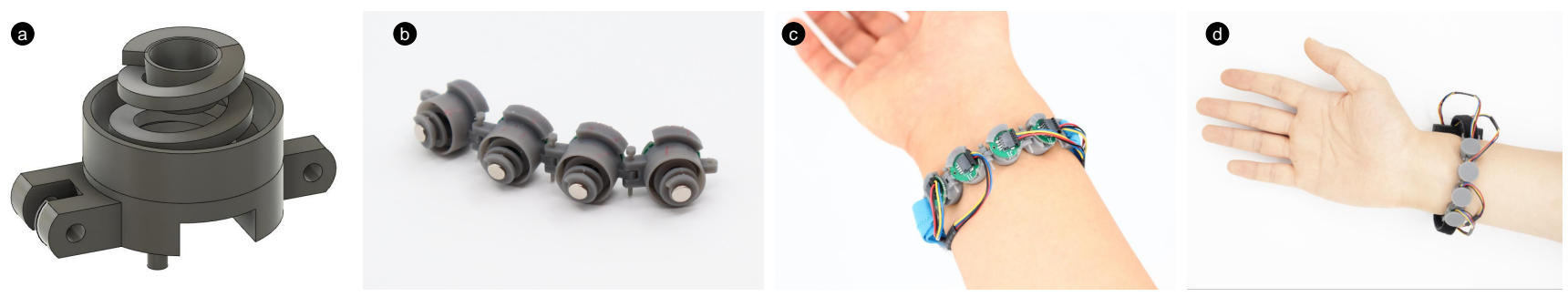

Figure 1: a) Sensor module design. b) Four sensor modules connect to form a bracelet. c) Bracelet prototype showing connections to the custom PCB. d) Final MyoSpring bracelet.

\begin{abstract}
We introduce MyoSpring, a novel technique to fabricate customised mechanomyographic sensors with 3D printed springs. MyoSpring averts from a traditional 'one-size-fits-all' approach and facilitates the development of functionally customisable wrist-worn sensors, which can be used to recognise subtle finger gestures with high accuracy. We automate the design process of MyoSpring sensors with an intuitive Graphical User Interface enabling novices to quickly design and fabricate customised sensors. We conducted a user study with 15 participants and 12 gestures, including partial and full flexion finger gestures, to verify the functionality of MyoSpring. Through the evaluation, we show that 1) MyoSpring can achieve high average accuracy of finger gesture sensing of $94.11 \%$ (SD = 9.73\%), 2) mechanical customisation allows MyoSpring to significantly improve the average accuracy of overall gesture sensing and 3) MyoSpring better supports partial finger flexion gesture sensing, achieving an accuracy of $91.44 \%(\mathrm{SD}=11.76 \%)$ for partial gestures.
\end{abstract}

\footnotetext{
*Also with The Department of Electronic and Telecommunication Engineering, University of Moratuwa, Sri Lanka.

${ }^{\dagger}$ Also with The University of Sydney Nano Institute (Sydney Nano), The University of Sydney, Sydney, NSW, Australia.
}

\section{(c) (7)}

This work is licensed under a Creative Commons Attribution International 4.0 License.

TEI '22, February 13-16, 2022, Daejeon, Republic of Korea

(c) 2022 Copyright held by the owner/author(s).

ACM ISBN 978-1-4503-9147-4/22/02.

https://doi.org/10.1145/3490149.3501321

\section{CCS CONCEPTS}

- Human-centered computing $\rightarrow$ Gestural input.

\section{KEYWORDS}

3D printed sensors, digital fabrication, personalisation, wearable gesture recognition, mechanomyography

\section{ACM Reference Format:}

Stephen Shiao-ru Lin, Nisal Menuka Gamage, Kithmini Herath, and Anusha Withana. 2022. MyoSpring: 3D Printing Mechanomyographic Sensors for Subtle Finger Gesture Recognition. In Sixteenth International Conference on Tangible, Embedded, and Embodied Interaction (TEI '22), February 1316, 2022, Daejeon, Republic of Korea. ACM, New York, NY, USA, ?? pages. https://doi.org/10.1145/3490149.3501321

\section{INTRODUCTION}

The dexterity of our hands allows us to perform fine finger movements, enabling us to make a variety of unique poses comfortably. Thus, using finger movements as a natural, subtle and intuitive input modality has become a prominent human-computer interaction paradigm in a variety of application areas including smart devices [43, 64], virtual and augmented reality $[14,18,22,32]$ and human-robot interaction [21, 78].

Due to the ubiquitous nature of wearable devices, researchers have focused on building finger gesture recognition systems with familiar form factors such as wrist bands or bracelets [44, 47], data gloves [4, 54], smart rings [71, 72], other finger-worn devices [59] and wearable skins [49]. Recently, more focus has been directed towards increasingly comfortable and non-obtrusive devices worn on 
the wrist or forearm, incorporating sensing technologies such as optical sensing [40], ultrasound [34, 80], electrical impedance [65, 82], electromyography (EMG) [47] and mechanomyography (MMG) [16, 44]. Among these methods, in ubiquitous applications, MMG sensors can be made in smaller form factors with reduced cost and good performance [17], while being less susceptible to electromagnetic noise [63].

Partial finger flexion can be used as a subtle interactive modality in public spaces where privacy is an important factor [66]. They are also relevant for people with motor impairments such as stroke, Parkinson's disease or arthritis, who may not have the full range of motion and strength in their tendons and joints to perform gestures like fully flexing their fingers [7, 20,30]. Despite the numerous technologies available, recognising partial finger gestures with wearable sensors remains a challenging problem as such gestures only incorporate minor finger movements when compared to full finger flexion. Therefore, recognition accuracy is highly sensitive to the signal quality of the sensors. Particularly, existing MMG sensors which often follow a 'one-size-fits-all' approach tend to lack the requisite mechanical sensitivity to deliver high-accuracy detection.

In this paper, we propose MyoSpring, a novel technique to fabricate a 3D-printed, wrist-worn, mechanomyographic sensor for subtle and partial finger gesture recognition. We introduce a design tool which allows even novices to quickly design MyoSpring sensors that can be fabricated with affordable stereolithography (SLA) 3D printers. To the best of our knowledge, this is the first attempt to develop a highly sensitive, customisable 3D-printed wrist-worn device capable of recognising partial finger flexion gestures. We evaluate our devices on 15 participants, using a gesture set containing both partial and full finger flexions. We show that our 3D-printed MyoSpring sensors can significantly improve gesture recognition performance, achieving $94.11 \%$ ( $\mathrm{SD}=9.73 \%$ ) classification accuracy across the users for the complete set of 12 different full and partial flexion gestures. An accuracy of $91.44 \%$ ( $\mathrm{SD}=11.76 \%$ ) is achieved when considering partial flexions alone.

Specific contributions of the paper are:

(1) A novel method of developing easily customisable mechanomyographic sensors with 3D-printed spring structures;

(2) A technical evaluation of our 3D-printed spring structures and a simplified data-driven design tool for generating 3Dprintable sensor designs;

(3) Results from a user study, demonstrating our solution leads to higher sensing accuracy and enables reliable partial finger movement detection to expand the traditionally available gesture vocabulary.

\section{RELATED WORK}

In this section, we will discuss the literature on finger gesture types, wearable gesture sensing technologies and customisation needs.

\subsection{Finger Gestures}

The dexterity of our hands allows us to perform fine hand and finger movements, which are extensively used with interactive devices today. Finger gestures can be considered in two aspects: on-surface gestures and on-air gestures. Wobbrock et al. [73] conducted an extensive classification of finger gestures for surface computing with 27 gestures. Wearable gesture sensing mainly focuses on on-air gestures, leveraging external and internal changes of the hand for recognition. Researchers have utilised various finger gestures for evaluating wearable devices, ranging from pinch, flick and snap gestures $[16,41]$ to finger taps and finger rubbing [70] and full flexion of fingers [47]. However, to the best of our knowledge, recognition of partial finger movements or partial finger flexions is not widely explored in the literature. Partial finger movements are subtle, making it a more challenging problem to recognise them with wearable devices.

Partial finger gestures enable a more subtle interaction modality for HCI when compared to other finger gestures. Interaction via subtle gestures is important to attract minimal attention in scenarios where other gestural input is socially awkward or privacyintrusive [38]. Additionally, people with disabilities, or motor impairments such as stroke or arthritis, may not have the capability to fully flex their fingers. Research has also indicated that because of the hand's physiology, gestures involving a slight flexion of the fingers are more comfortable than those requiring adjacent fingers to be 'discordant', i.e. one finger fully flexed whilst the other is extended [56]. These partial finger gestures are identifiable with vision-based techniques, including commercial devices such as VR headsets ${ }^{1}$ and LeapMotion ${ }^{2}$. However, vision-based techniques are limited due to occlusions and privacy concerns.

\subsection{Finger Gesture Recognition with Wearable Sensors}

Wearable hand gesture recognition systems come with various form factors including gloves [12, 48], rings [71, 72] and wrist-worn devices $[34,40]$. However, less obtrusive devices such as wristbands and smart watches hold advantages in aesthetic appeal and practicality. Vision-based methods, using optical and infrared (IR) sensors mounted on the wrist, detect the geometrical changes of the hand when a gesture is performed [26, 31, 40]. These geometrical changes can also be measured with ultrasonic sensors $[34,80]$, using reflected signals of the emitted acoustic waves for gesture recognition. Similar to these geometrical changes of the hand, internal changes in the hand can be used for finger gesture recognition. Active sensing methods, such as electrical impedance-based techniques, use emitter-receiver pairs to excite the hand and measure the change in bio-impedance for gesture recognition [55, 65, 82]. Passive sensing methods such as EMG measure the electrical signals generated by muscle activity [47, 81]. However, EMG-based gesture recognition is more susceptible to electromagnetic environmental noise [63].

In contrast to the above-mentioned sensing techniques, MMG involves detection of the low-frequency vibrations created by muscle contractions and oscillations, also known as bio-acoustic signals or "muscular sounds" [52, 74]. These vibrations can be captured by inertial sensors such as accelerometers [41, 44] and microphones $[3,61]$ with wrist-worn wearable devices for finger gesture recognition. Additionally, pressure and force sensors can be used to measure changes in the shape of the contracting muscles, which is also known as Force Myography (FMG). Researchers have

\footnotetext{
${ }^{1}$ https://www.oculus.com/

${ }^{2}$ https://www.ultraleap.com/product/leap-motion-controller/
} 

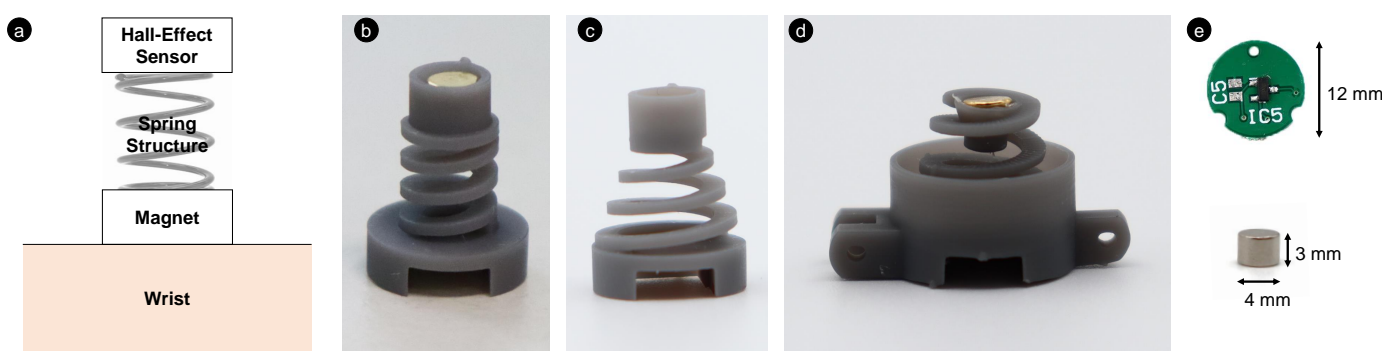

Figure 2: a) Conceptual design of our mechanomyographic gesture sensor. b) Early prototype with cylindrical spring. c) Prototype using conical spring. d) Redesigned conical spring with cylindrical wall. e) Custom PCB with TI-DRV5055A1EDBZRQ1 Hall-Effect Sensor and Eclipse N802RS 4 mm x 3 mm Neodymium magnet.

shown that FMG can successfully be used in finger gesture recognition $[5,16,37,60]$.

\subsection{Customisation Needs}

Gestures are personal. Individuals perform gestures differently [34], with varying ranges of speeds, orientations, postures and unique inherent characteristics. Due to these variations, software customisation such as personalised machine learning models perform better in human gesture recognition [6] than generalised machine learning models. This also holds for finger gesture recognition, where personalised machine learning models are used to improve gesture recognition accuracy $[34,41,82]$.

Advancements in rapid fabrication techniques have enabled HCI researchers to explore the fabrication of customised devices and interfaces in aesthetics and form factor [28, 69]. For example, Kawahara et al. [39] proposed conductive inkjet printing to quickly and easily develop customised electronic circuits which are used by many researchers today for novel interactive devices [49, 53]. Further, researchers have devised techniques for creating functionally customisable interactive devices, including taking an additional step to developing a complete digital fabrication pipeline [51, 57, 58, 77]. The customisation of mechanical functions with $3 \mathrm{D}$ printing is introduced by Ion et al. [33] allowing users to design and fabricate mechanically functional objects such as door latches and pliers. However, functional customisation of wearable sensors is a relatively unexplored topic. To address this, MyoSpring proposes a method for customisation of gesture recognition sensors, in turn allowing sensor sensitivity to be modified according to user preference.

\section{MYOSPRING}

To mitigate the intrusiveness of hand- or finger-worn devices, research shows that finger movements can be accurately sensed using muscle and tendon movements of the forearm [42, 46]. Particularly, the wrist provides a promising location to sense finger movements since it is a familiar location for wearables such as a wristwatch [42], and also a majority of tendons and muscles that connect to the fingers run through the wrist through the carpal tunnel. The mechanical vibrations at the carpal tunnel caused by finger movements can be captured using pressure, piezo or inertial sensors attached to the skin surface, which have been shown to accurately recognise hand activity [5, 37].

The construction of each individuals' wrist can differ significantly in size, cartilage and tissue structures, resulting in different viscoelastic properties on the carpal tunnel. Even within the same person, properties such as skin elasticity are known to decrease profoundly with age [25]. These changes in mechanical properties greatly affect the signal qualities acquired by a mechanical sensor. However, designing a sensor to match individuals is a challenging task and material choices to simulate different mechanical properties are limited [37].

Alternatively, customised mechanical structures can be used to match tissue characteristics to sensors. Advances in additive manufacturing processes provide an easier, faster and safer means to design and fabricate physical structures with varying mechanical properties [29, 33, 39, 50, 57, 58]. Particularly, compression spring structures provide a great opportunity and can be 3D printed economically with customisable behaviours [29]. However, in a wearable context, these structures need to be embedded in a much smaller form factor than shown in this previous research (e.g. 1.6 $\mathrm{mm}$ wire thickness and $3.6 \mathrm{~mm}$ diameter in [29]) and the parameters to fine-tune their mechanical property such as spring constant ( $k$ of Hooke's Law) need to be carefully chosen while minimising physical size. In this paper, we present MyoSpring, a novel method of developing mechanically customisable mechanomyographic sensors which can be easily and economically $3 \mathrm{D}$ printed using a commodity $3 \mathrm{D}$ printer.

\subsection{Design and Fabrication Process}

We followed an iterative prototyping process in developing MyoSpring, following three design goals in our design and implementation.

3.1.1 Form factor: Our first goal is to determine the feasibility of printing spring structures to fit a wearable form factor. In our study, we designed springs with various sizes (diameter $6 \mathrm{~mm}$ to $8.5 \mathrm{~mm}$ and height $7 \mathrm{~mm}$ to $15 \mathrm{~mm}$ ) then printed them using commonly available 3D printers and materials (Ultimaker S5 with PLA, TPU and PVA filament and Formlabs Form 2 with Grey v4 resin). While previous research shows the Ultimaker S3 3D printer and Tough PLA + PVA material can be used to successfully print springs [29], at small sizes, the majority of them failed due to mechanical instability, poor printing quality and obstructions from the support material. 

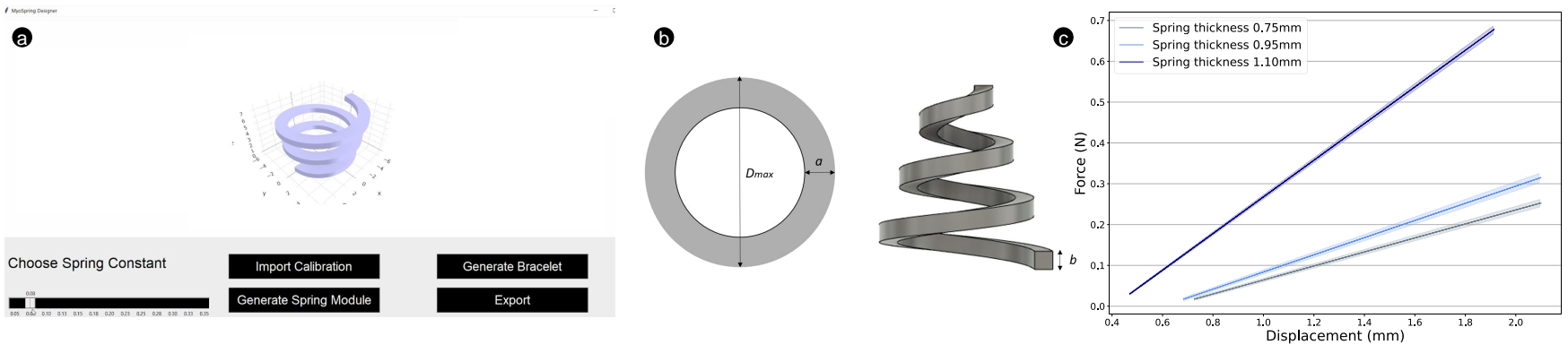

Figure 3: a) GUI interface of the design tool. b) Conical shaped spring with a rectangular cross-section wire c) Results from empirical study, showing how coil thickness $b$ effects the spring constant $k$.

The consistent exception in our observation was the Formlabs Form 2 with Grey v4 Resin, where high resolution of the printer and less support material helped us to print high quality springs.

3.1.2 Sensing Mechanism: Our second goal is to find an effective sensing method to measure the spring mechanics. Several possible sensing choices are available for capturing the vibrations of the spring structures. Intuitively, attaching an IMU, piezo or resistive pressure sensor on the spring could be used to sense spring movements. However, these need additional electrical connectivity across the spring, which in turn will affect the spring properties. We also considered using capacitive sensing with a grounded electrode at the end of the spring, however, capacitive sensing works with a much smaller range at high resolutions than the printable spring designs $[62,68]$. It is likely that the distance from the sensor to the passive plate would exceed this range, meaning the sensor would be unable to detect subtle tendon movements [67]. Therefore, we chose Hall effect sensing with a small passive magnet at the end of the spring as a viable solution. As shown in Figure 2-a, the magnet can be attached at one end, while the Hall effect sensor and the electronics can be placed at the other end. This approach did not require any electrical wiring across the spring and works very well with the range of the spring. More importantly, Hall effect sensors have a much higher bandwidth (up to $20 \mathrm{kHz}$ ) and consume a small amount of power $(3.3 \mathrm{~mW})^{3}$, and do not get affected by proximity to the body.

3.1.3 Spring Stability: Our third goal is to design a stable physical structure to accommodate device movements. The cylindrical springs (Figure 2-b) were unstable and would bend or buckle during compression. To improve stability, as shown in Figure 2-c, we designed conical springs with an angle of $15^{\circ}$, which significantly reduced buckling as their non-uniform coil diameter effectively decreases the spring's slenderness ratio $[8,76]$. Finally, a cylindrical wall of height $4 \mathrm{~mm}$ was added around each spring to reinforce the springs' compression behaviour parallel to their central axes (Figure 2-d). Attachment points were added on each side to enable a modular design whereby individual springs could be connected and disconnected to form a wearable bracelet.

\footnotetext{
${ }^{3}$ Texas Instruments TI-DRV5055
}

\subsection{MyoSpring Design Tool}

Designing MyoSpring sensors can be a challenging task for a novice user as it requires careful manipulation of parameters to design a spring with a given spring constant. Additionally, material-specific parameters are not readily available for springs, making it harder to choose the spring parameters suitable for a given spring constant. Designing other parts of the sensor, including the intricate details that should be considered when constructing the complete device, takes a meticulous effort. We have experienced poor designs leading to structural failure, which emphasises the need for fine tuning of the design. To overcome these challenges, we automate the design process of MyoSpring sensors with a simplified design tool. Using the Cadquery ${ }^{4}$ Python library in the backend, our tool allows users to modify the spring design using a simple Graphical User Interface (GUI) as shown in Figure 3-a. With this tool, users can mechanically customise the stiffness of the sensor by changing the cross-sectional dimensions of the spring through a simple slider in the GUI. After the desired spring stiffness is selected, the tool generates all the 3D design parts including the additional parts required for assembly, which are all exported as 3D-printable STL files. The user input will only modify the spring stiffness while other parts are automatically generated using pre-designed models inbuilt in the tool.

Our designed tool was developed with the results of several empirical studies we performed to evaluate the behaviour of the printed springs. For a conical spring with a rectangular crosssection wire, the spring constant is given in Equation 1 where $a$ and $b$ are dimensions of the cross-section, $G$ is the modulus of elasticity in shear, $\epsilon$ is the shape coefficient, $n$ is the number of coils and $D C$ is a value which depends on the maximum and minimum diameters of the spring ${ }^{5}$ as shown in Figure 3-b. Through our iterative prototyping process, we discovered that changing the thickness of the coil is the optimal way of manipulating spring designs in order to control spring constant, whilst maintaining a relatively uniform form factor. Therefore, we can infer that $k=A b^{2}$ where $A$ is a constant that depends on the other physical parameters of the spring and material properties. Our evaluations show that our empirical data from the compressive test closely follows the theoretical results as shown in Figure 3-c with $R^{2}=.96$. We utilise this relationship in our design tool and approximate the constant

\footnotetext{
https://cadquery.readthedocs.io/

${ }^{5}$ https://www.mitcalc.com/doc/springs/help/en/springstxt.htm
} 

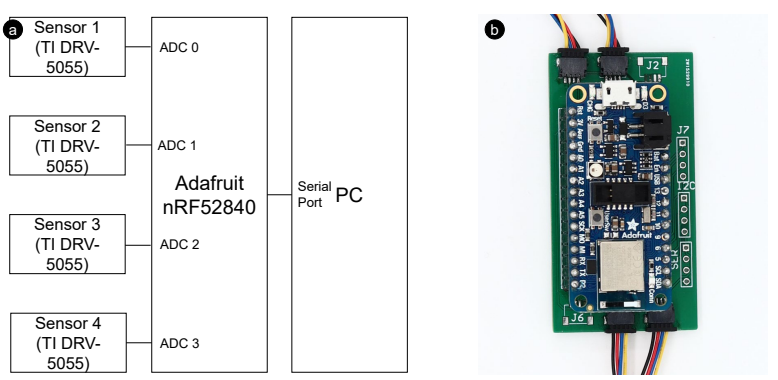

Figure 4: a) Diagram of hardware setup. b) Adafruit nRF52840 board, to which the four sensors were connected via custom PCBs.

value $A$ with compression and force measurements from a single reference spring, such that any subsequent springs can be designed as similar to the desired spring constant as possible.

$$
k=\frac{4 G a^{2} b^{2}}{\epsilon n \cdot D C}
$$

We performed compressive testing of our springs with an Instron 3367 Tensile Test machine for 10 cycles. We observed that our theoretical results closely follow the empirical results. All three of our springs followed a linear relationship between the compressive force and the displacement for our measurement range (Figure Figure 3-c), as supported by the conclusions drawn by $\mathrm{Wu}$ and $\mathrm{Hsu}$ [76]. We then approximate the spring constant of the above three springs in the linear range as $0.179(\mathrm{SD}=0.002), 0.217(\mathrm{SD}=0.003)$ and $0.449(\mathrm{SD}=0.003) \mathrm{N} / \mathrm{mm}$ respectively.

To evaluate whether mechanical properties have an effect on the sensitivity and gesture recognition accuracy of MyoSpring sensors, a series of sensors with different spring constants should be compared. Following a pilot study with two users to find the hardest and softest springs that can produce reliable signal, we selected three springs: soft $(0.75 \mathrm{~mm}$ thick $)$, medium $(0.95 \mathrm{~mm}$ thick $)$ and hard $(1.10 \mathrm{~mm}$ thick). We limited the spring factor levels to three to reduce the experiment time and avoid participant fatigue.

\subsection{Electronics and Data Collection Hardware}

Upon finalising the spring designs, a wearable bracelet was constructed by connecting four spring modules, each with a $4 \mathrm{~mm} \times$ $3 \mathrm{~mm}$ neodymium disc magnet attached (Figure 1-b). As shown in Figure 1-c,d, the four sensors were spaced $16.5 \mathrm{~mm}$ apart from each other to evenly cover the underside of the average wrist, as it has been shown that having sensors on the other side of the wrist would be redundant [37]. For each sensor module, a Texas Instruments DRV-5055 surface-mount Hall-effect sensor was attached to a custom-designed printed circuit board (PCB) as depicted in Figure 2-e. Each PCB was then connected to an Adafruit Feather nRF52840 Express microcontroller board, shown in Figure 4-b. For our evaluation, we connected the microcontroller to a PC's serial port and recorded data at $340 \mathrm{~Hz}$, as previous research indicated the bandwidth of muscle vibrations is generally below $140 \mathrm{~Hz}$ [52], while [74] suggested MMG techniques should measure muscle oscillations in the range $2-200 \mathrm{~Hz}$. The $\mathrm{nRF}$ board includes a Bluetooth

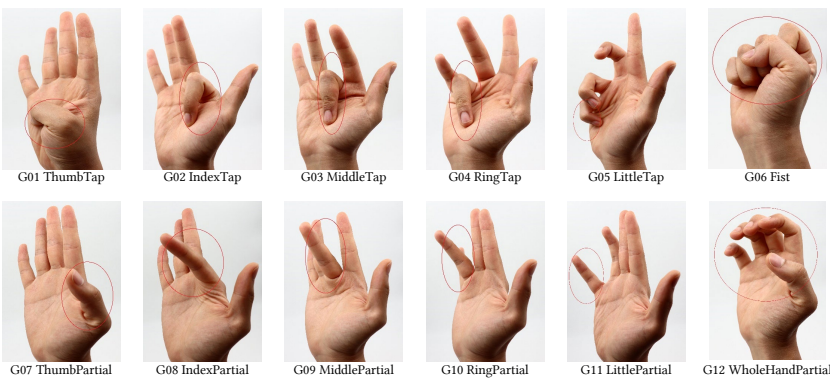

Figure 5: Gesture set used in our evaluation. G01 to G06 are full finger flexion gestures, others are partial.

Low Energy module ${ }^{6}$ which may be of use in future research in constructing a portable wireless prototype. The final bracelet prototype weighed $48 g$ excluding the USB cable.

\section{EVALUATION}

To evaluate the performance of MyoSpring as a gesture recognition device, we conducted a controlled user study with 15 participants. We aim to answer three questions through this user study: 1) Can 3D printed spring-based mechanomyographic sensors accurately detect finger gestures? 2) Does the mechanical customisation of spring properties result in superior sensor performance? and 3) Do the mechanically customised spring sensors perform better at recognising partial finger flexions?

\subsection{Gesture Selection}

To evaluate MyoSpring, we selected a gesture set with both full and partial finger flexions. The reduced amplitude of partial finger flexions is harder to detect, yet these movements are also easier for certain individuals to perform such as those with motor impairments. Additionally, we include the full finger flexion gestures in the gesture set to compare against partial finger movements and the literature. Figure 5 shows the selected gesture set. G01 to G06 represent full finger flexions, and G07 to G12 represent partial finger flexions. The photographs in the figure depict the images used in the user study GUI setup. Individuals performed each gesture with their own variations of range. However, the experiment conductor carefully observed for partial finger movements that exceed half of the full flexion range and asked the participant to repeat in such cases.

\subsection{Experiment Setup and Procedure}

The study followed a $3 \times 12$ factorial design with the three bracelet types described in Section 3.2 (soft, medium and hard) and the selected 12 finger flexion gestures. The experiment was divided into three blocks, in each block the participants wore one bracelet type and performed the gestures. The order of blocks (i.e. bracelets) was counterbalanced using a Latin square. Within the block, a randomly selected gesture was presented one at a time for the user to perform, such that each gesture would eventually appear 10 times in total. This resulted in $3 \times 12 \times 10=360$ gesture iterations

\footnotetext{
${ }^{6}$ https://www.adafruit.com/product/4062
} 

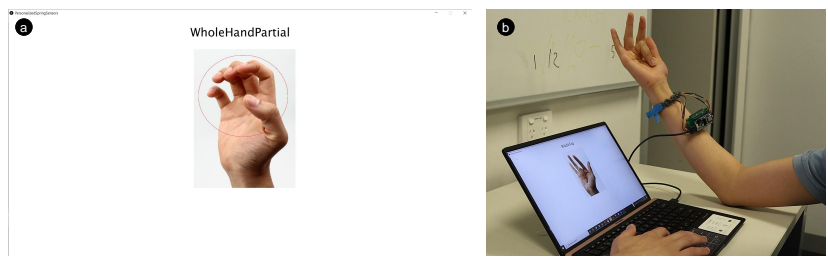

Figure 6: a) GUI used for the user study. b) Performing a gesture during the experiment.

per participant and ensured there would be minimal correlation between samples, to better reflect a real-life use case where users would be unlikely to perform the same gesture multiple times in a row.

The study was conducted with express ethics approval from the University and followed COVID-19 safety protocols such as wearing a face mask, cleansing all equipment pre- and post-study and social distancing. We recruited 15 healthy adult participants (6 female, 2 left-handed, mean $_{\text {age }}=24.67$ years, $\mathrm{SD}_{\text {age }}=4.82$ ). Following the formal procedures such as consent forms and collecting background data, each participant was briefed about the project and the study tasks, and given trials to become familiar with the setup. They were informed that they could opt out of the study anytime during the experiment. During the experiment, the participant was seated, the bracelet was attached to the wrist of the participant's dominant hand, and they supported the hand by resting their elbow on the table (Figure 6-b). In order to ensure that all three sensor wristbands were attached in an identical location between blocks, small segments of D3 kinesiology tape were attached to the participant's wrist to indicate and align the position of sensors. Between each block, participants were given a five-minute break to alleviate fatigue.

During each block, as shown in Figure 6-a, a simple Graphical User Interface (GUI) showed the name and an image of the finger gesture to be performed by the participant. After completion, the next gesture was presented after a brief interval. Performing a gesture incorrectly (for example, performing a partial flexion gesture when a full flexion is required, or using a different finger than indicated) would require that gesture to be re-performed. The study was conducted using an ASUS UX433FN laptop (Intel Core i7-8565U, 1.9 GHz, $16 \mathrm{~GB}$ RAM) and data was recorded at $340 \mathrm{~Hz}$ per each sensor (i.e. $340 \mathrm{~Hz} \times 4$ per bracelet). In addition to the digitised values from each sensor, the trial number, block number, redo counter, timestamp (ms), gesture identifier and iteration number were recorded. A post-study questionnaire was then administered to gain subjective feedback about the comfort of the sensor, ease of performing the gestures and whether the participant had additional comments or suggestions.

\subsection{Data Preprocessing}

We observed that signals from each sensor had a large DC shift. This was mainly due to the degree of compression that each sensor of the bracelet was subjected to when the bracelet is fastened to the users' wrist. Therefore, this shift would vary slightly for each participant and each experiment block, based on the tightness of the bracelet on the users' wrist. In order to avoid this factor affecting the classification process, the DC shift was removed from each signal.

While past work has often performed windowing by splitting the data into predefined constant time intervals [5], this was not an optimal approach for our data given we observed significant variance in the amount of time taken for each participant to perform a gesture. Therefore, we implemented a windowing algorithm by considering the time windows or contiguous 'slices' of the signal during which the data exceeded or fell below a predefined threshold of mean $+3 \times S D$, with the mean and standard deviation calculated from data recorded while the user was stationary prior to commencing each gesture iteration. The starting point of a gesture was taken to be the initial index of the first $50 \mathrm{~ms}$-long slice during which any of the sensor's measurements exceeded the threshold. In a similar manner, the end point was recorded as the first index of the first $50 \mathrm{~ms}$ slice during which all measurements from any sensor fell below the threshold. This guarded against the possibility of the window being incorrectly cut off where the signal briefly fell outside the threshold at any point while performing the movement.

To ensure robust classification results, feature selection was then done in two separate stages. The first was using the tsfresh Python framework, which automates the selection of significant attributes from the time series [11]. The default false discovery rate (FDR) threshold of 0.05 was chosen to select features from each dataset. Upon obtaining the tsfresh features, we imported the generated features into the Weka machine learning tool, where further attribute selection was conducted to reduce the amount of data used for classification. Specifically, the 'CfsSubsetEval' package was used together with the GreedyStepwise ranking method. CfsSubsetEval uses the Correlation-based Feature Selection method to select subsets of features that are highly correlated with the class while having low intercorrelation [27]. As a result, this tended to increase classification accuracy during pilot studies, while also improving the time taken to perform the classification task which is desirable for a real-time application. On average 80 features were extracted for each user. Features such as absolute energy, longest strike below mean, minimum value, Benford correlation, first location of minimum, kurtosis, skewness and the first, second and the third Fourier coefficients were some of the features that were selected through this process. Of these, skewness, kurtosis, minimum value, the first location of minimum and longest strike below mean provide information relating to the envelope or distribution of time series data. Moreover, Benford correlation provides information on the pattern of the time series data. In the context of signals captured by MyoSpring, the absolute energy provides a measure of signal strength. Furthermore, it can be observed that the selected Fourier coefficients capture useful frequency domain information of the signals. Therefore, the features extracted by the proposed feature selection process effectively capture the time, frequency and statistical characteristics of time series data.

4.3.1 Classification. After preprocessing and feature selection, classification was conducted using the Weka framework for machine learning. For the pilot study, the K-Nearest Neighbour, Naïve Bayes, Simple Logistic Regression, Sequential Minimal Optimisation (SMO) and Random Forest algorithms were chosen and then 

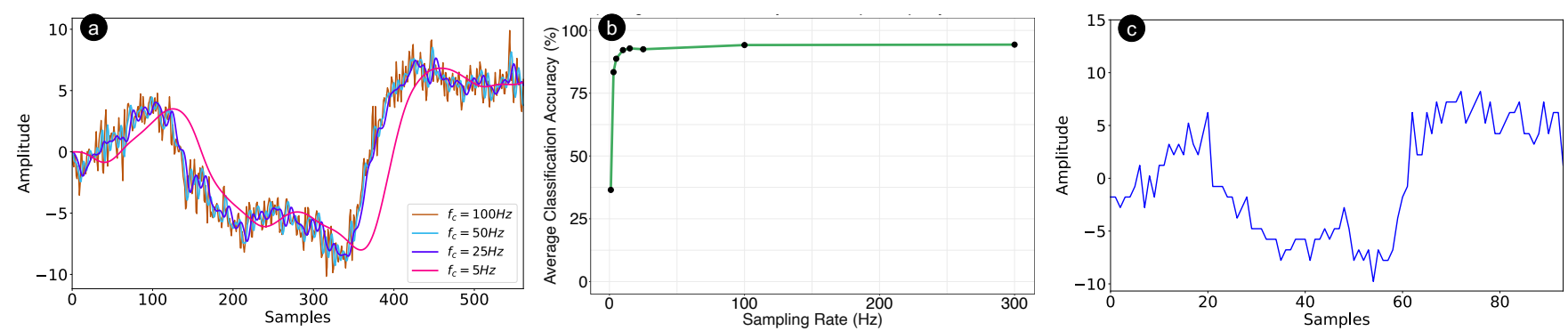

Figure 7: a) Low-pass filtered versions of the original signal from a sensor (a randomly selected gesture). b) The effect of reducing downsampling frequency on the average classification accuracy of the best bracelet. c) Signal downsampled at $50 \mathrm{~Hz}$.

run in Weka using 10-fold cross-validation, for classification of each movement from the reduced data set for each individual user. Splitting the limited amount of data into train and test sets (usually an 80-20 split) cannot lead to realistic conclusions as the test set will be limited to 2 examples per gesture. This can lead to any performance outcome purely due to chance. Therefore, as with similar prior work, a 10-fold cross-validation approach was used to reduce the effects of overfitting, and it is suitable for evaluating how the models would work with unseen data [5, 36, 45, 47, 83] We observed that SMO achieved the most consistent classification performance, compared to the other algorithms which tend to exhibit a higher degree of variability. Therefore, we decided that SMO would be used for classification and for reporting accuracy scores in the final study. Our tests showed that for real-time classification with a pre-trained model, SMO was able to classify a gesture in $48.16 \mathrm{~ms}$ on average (SD: 27.03ms) with a Dell OptiPlex 7070 PC (Intel Core i7-8700, $3.2 \mathrm{GHz}, 32 \mathrm{~GB}$ RAM).

4.3.2 Frequency Analysis. When we obtained the frequency spectrum of each signal captured by the four sensors, it was evident that the strongest frequency components of the signals belonged to the frequency region less than $15 \mathrm{~Hz}$. As shown in Figure 7-a, when we applied a low-pass filter to the original signal at cutoff frequencies as low as $50 \mathrm{~Hz}, 25 \mathrm{~Hz}$ and $5 \mathrm{~Hz}$, we observed that the filtered signals retained a good abstract envelope of the information. Therefore, we conducted an experiment on downsampling the signal at the software level for frequencies within the range $1 \mathrm{~Hz}-100 \mathrm{~Hz}$. For each downsampled instance, we classified each block using SMO. Then we identified the best, middle and worst performing bracelets per user based on the classification accuracies for each downsampled instance. Figure 7-b shows the average classification accuracies for each sampling frequency's best-performing bracelet. We observed that the average classification accuracy of $92 \%$ at $10 \mathrm{~Hz}$ sampling rate began to drop significantly as the sampling frequency was reduced further (Figure 7-b), indicating that our system is still capable of high classification accuracy even at a sampling frequency as low as $10 \mathrm{~Hz}$. Therefore, it will facilitate reducing the hardware complexity of the analog to digital converter. Furthermore, reducing sampling frequency guards against the risk of the signal being impacted by external noise caused by gravity or minute changes in the device's orientation, as high frequency components $(\geqslant 50 \mathrm{~Hz})$ will be filtered out in this process. As the maximum achievable average classification accuracy for the best bracelet begins to reduce around
$50 \mathrm{~Hz}$, signals resulting from the $50 \mathrm{~Hz}$ sampling frequency were used for further analysis (an example signal is shown in Figure 7-c).

\subsection{Results}

As described in Section 4.3, we windowed the time domain data, used the tsfresh library to calculate features and used the SMO Support Vector Machine (SVM) machine learning algorithm from the Weka library to calculate the classification accuracies of each gesture. We discuss the results along the three aims of the user study. The Shapiro-Wilk test revealed that accuracies were not normally distributed and thus all statistical analysis was conducted with the Friedman non-parametric test and post-hoc Wilcoxon signed-rank tests. All tests were conducted in R.

4.4.1 Overall Gesture Accuracy. Results show that the 3D-printed MyoSpring sensors can detect combined full and partial finger gestures with a high average accuracy of $94.11 \%$ (SD $=9.73 \%$ ) across the fifteen participants (Figure 8-a All Gestures). This performance is comparable to that achieved by other wearable finger gesturerecognition solutions which do not include partial finger movements in their gesture sets (e.g. 95\% with 10 participants [83] and $94 \%$ with 12 participants [47]). Considering the six partial finger flexions alone, our MyoSpring sensors were able to achieve an average accuracy of $91.44 \%$ (SD $=11.76 \%$ ). Thus, these results indicate that MyoSpring is able to achieve similar or better accuracy results in comparison to prior work, despite recognising more challenging partial finger gestures.

4.4.2 Effect of Customised Springs. The type of spring which performed best varied for different participants (soft - 5 participants, medium - 6, hard - 4). This is an early indication that mechanical customisation leads to better performing sensors. Further analysis of overall gesture classification accuracy data with the Friedman non-parametric test showed that the type of spring used for the bracelet had a statistically significant effect $\left(\chi^{2}(2)=49.634\right.$, $p=1.668 \mathrm{e}-11)$. The post-hoc Wilcoxon signed-rank test showed a statistically significant difference in average classification accuracy between bracelet pairs worst-middle $(Z=-3.287, p=0.001)$, middle-best $(Z=-3.856, p=0.0001)$ and worst-best $(Z=-6.466, p=$ $1.01 \mathrm{e}-10)$. This shows a significant improvement of average gesture recognition accuracy across all participants when selecting the best bracelet for each user. It is important to note that even comparing 

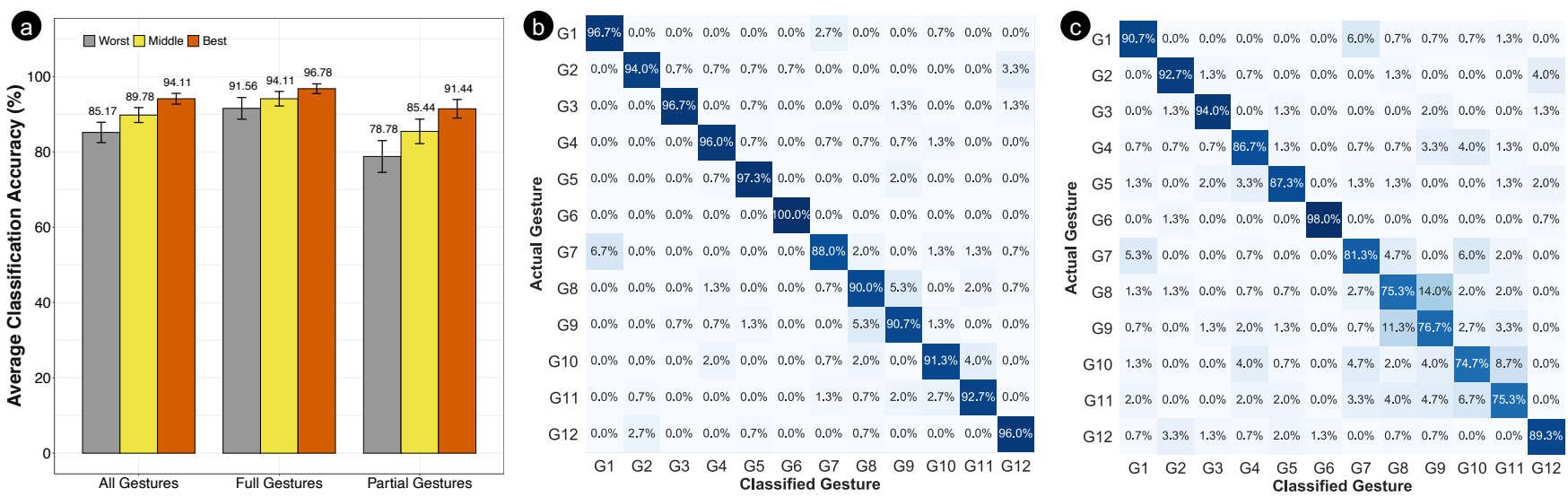

Figure 8: a) Average classification accuracies for a participants' worst, middle and best bracelet considering all, full and partial gestures. b) The confusion matrix for the best-performing bracelet of every user. c) The confusion matrix for the worstperforming bracelet of every user.

the middle-performing bracelet to the best-performing showed significant improvement ( $89.78 \%$ to $94.11 \%$ ), which further asserts the importance of mechanical customisation.

4.4.3 Customisation and Partial Finger Gesture Detection. Subgroups Full Gestures and Partial Gestures in Figure 8-a, contrast the effect of customisation on full and partial finger flexion recognition. Analysis of data with the Friedman non-parametric test considering only the partial gestures showed a statistically significant effect of the bracelet type $\left(\chi^{2}(2) 40.142, p=1.92 \mathrm{e}-09\right)$. Post-hoc Wilcoxon signed-rank test showed a statistically significant difference in average classification accuracy between bracelet pairs worst-middle $(Z=-3.186, p=0.001)$, middle-best $(Z=-2.908, p=0.0036)$ and worst-best $(Z=-5.381, p=7.41 \mathrm{e}-08)$. Importantly, we note the accuracy drop between full and partial gestures for worst $(91.56 \%$ to $78.78 \%$ ) and medium bracelets $(94.11 \%$ to $85.44 \%)$, both around a $10 \%$ reduction in accuracy. However, for the best bracelet, the drop in accuracy, while significant, was less than 6\% (96.78\% to $91.44 \%$, Wilcoxon signed-rank test $Z=-3.754, p=0.0002)$. We can further explain these results by looking at the confusion matrices as shown in Figures 8-b,c. For the worst bracelet (Figure 8-c), partial finger gestures (G7 to G12) show a much higher confusion compared to the same in the best bracelet (Figure 8-b). It can be seen that for the worst-performing bracelet, the majority of classification confusion arises between the partial finger flexion gestures involving the index, middle and ring fingers (G08-G11), for example the index finger gesture was misclassified as the middle finger gesture $14 \%$ of the time. This high degree of confusion is avoided when using a bracelet setup with a functional design more suited for that user. In other words, this proves mechanical customisation of sensing devices is critical for accurate partial finger gesture recognition.

Furthermore, Figure 9 shows the difference in the quality of the resulting waveforms when the best bracelet is used, compared to the middle and worst bracelet. The difference is observed to be significant for the waveforms resulting from partial gestures, where the best bracelets show a higher variation in amplitude while the waveforms from the middle and worst bracelet show relatively low variations in amplitude. This further justifies the significant difference in average classification accuracies reported for partial gestures between the bracelet types in Figure 8-a. It can be concluded that the best 3D-printed MyoSpring for a user is capable of capturing a superior signal, and the performance is significantly improved for partial finger gestures.

\section{EXAMPLE APPLICATIONS}

In this section, we describe two example application scenarios for MyoSpring: a subtle interaction mode for controlling a mobile smart device, and a music-based rehabilitation game.

\subsection{Discreet Gesture Interaction}

In crowded settings or public spaces, users may feel uncomfortable while using a smartphone, tablet or other mobile device. Personal information such as a PIN, caller ID, or even the title of the currently playing song can be visible to anyone standing in close proximity to the screen. Alternatives to using touch interaction, such as voice control assistants like Apple's Siri, suffer from similar limitations regarding privacy $[1,19,23]$. Additionally, using voice interaction is not ideal in either silent locations (for example, libraries), or in noisy settings such as public transport.

Hence, we propose and demonstrate the use of MyoSpring to enable subtle control of such mobile devices in public settings. A user can assign partial finger flexion gestures to commands such as playing and pausing media, switching between music tracks or adjusting the volume, allowing them to control their phone eyesfree whilst keeping the device in their pocket as shown in Figure 10a,b,c. Furthermore, users can map finger gestures to core functions of the smartphone such as rejecting calls. This form of almostimperceptible control can help reduce disruptions and distractions, for example during a meeting or presentation situation.

\subsection{Music-Based Rehabilitation}

Past work has shown the effectiveness of Therapeutic Instrument Music Performance (TIMP) on improving the joint function and 

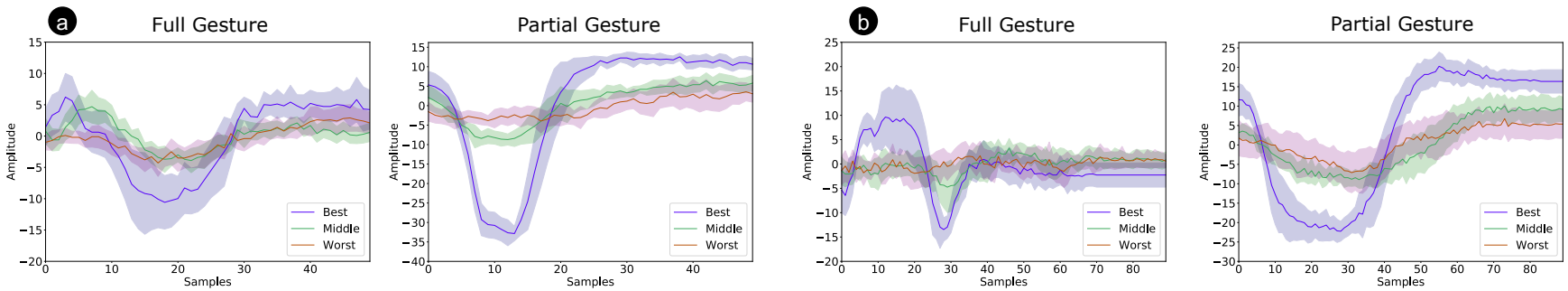

Figure 9: Comparison of the averaged waveforms of the best, middle and worst bracelets per gesture per user. a) and b) depicts the averaged waveforms from a single sensor from the best, middle and worst bracelets of 2 gestures from 2 randomly selected users.

coordination of people with cerebral palsy [2,9], multiple sclerosis [24], osteoarthritis [79] and stroke [10, 35]. However, not all individuals have convenient access to a musical instrument such as a piano. Even if they do, they may lack sufficient finger strength to operate it [13]. Other applications which implement TIMP without requiring an actual musical instrument use technologies such as haptic gloves [35], which can also be prohibitively expensive or unfamiliar to users.

We propose that our MyoSpring device can enable a user to perform finger rehabilitation exercises without the need for further specialised equipment. In doing so, users can improve their finger fractionation (independence) in an accessible and engaging manner. Furthermore, as our device can recognise varying degrees of partial finger movement, the application can train the user's finger speed and strength by instructing them to play notes louder (with more force) or softer. Thus, it provides additional flexibility and utility compared to traditional means of finger rehabilitation, whilst importantly being entertaining and accessible to users.

Our application is implemented as a Graphical User Interface (GUI) created in Processing 3 as shown in Figure 10-d,e. A therapist or the user themselves can input a sequence of finger numbers in the left and right hand which can match any musical piece. The corresponding piano keys on the screen are highlighted in blue, one after the other. When the user moves the correct finger, the key changes colour to green and the note corresponding to that key is played to provide auditory feedback. At the conclusion of the application, detailed feedback on the user's performance can be displayed.

\section{DISCUSSION}

The results of our study provide insights into the importance of the customisation of sensors for a wearable gesture-recognition solution, and also the applicability of our solution to the task of recognising a range of finger gestures including partial finger flexions. In this section, we will discuss these insights in greater depth and point out other observations of interest.

\subsection{The Importance of Mechanical Customisation}

Our results indicate that mechanical customisation of sensors leads to higher classification accuracy for partial finger gestures. This is evident by analysing the difference between the classification accuracy of each participant's best- and worst-performing bracelet. We found that on average, a participant's best-performing bracelet achieved overall gesture classification accuracies $8.94 \%$ higher than their worst-performing bracelet, and $12.67 \%$ higher when considering partial gestures only. This is a significant difference equivalent to an additional eleven gesture iterations being classified correctly out of the 120 iterations tested per bracelet, per user. Furthermore, through the classification confusion matrix shown in Figure 8-b,c, it is evident that customisation significantly contributes to reliable partial finger gesture recognition by reducing the amount of confusion between similar movements.

6.1.1 Mechanical relationship between anthropometric characteristics and spring stiffness. Although we have shown that a customised bracelet delivers higher gesture recognition accuracy than a noncustomised bracelet, a further question remains with regards to the most suitable methodology for selecting the optimal spring constant for a given user. We found there was no correlation between either BMI and optimal spring constant $\left(R^{2}=0.302\right)$, or wrist circumference and optimal spring constant $\left(R^{2}=0.261\right)$. To gain further insights regarding how spring-based mechanomyographic sensors should best be personalised to individual users, we conducted a follow-up study on three of the original 15 study participants to measure their skin and tissue elasticity and forces generated by carpal tunnel tendons when they perform finger gestures. To choose the three participants, we selected one who achieved the highest accuracy with the soft springs (P01), one with the medium (P11) and one with the hard springs (P10). We instructed each participant to place their hand below a Starr FGD-50 digital force gauge mounted to a test stand with a built-in $0.01 \mathrm{~mm}$ digital scale. We measured the force required to compress their skin by 1,2 and $4 \mathrm{~mm}$ at both the centre and side of the wrist. Subsequently, participants were asked to perform a series of partial and full finger flexions, while the force gauge recorded the amount of exerted force at the same wrist locations at depths of 2 and $4 \mathrm{~mm}$.

From the results of the follow-up study, we can anecdotally establish a relationship between the force measurable at the wrist during finger movements, and the most suitable spring stiffness to achieve optimal classification accuracy. For example, all three participants' wrists had similar $k$-values $(k=0.30,0.32,0.33 \mathrm{~N} / \mathrm{mm})$, yet participant $\mathrm{P} 01$ exerted on average less than half as much force $(0.46 \mathrm{~N})$ during finger movements compared to P10 (1.34N). Hence, the soft springs were more suited to $\mathrm{P} 01$ while hard springs recorded 

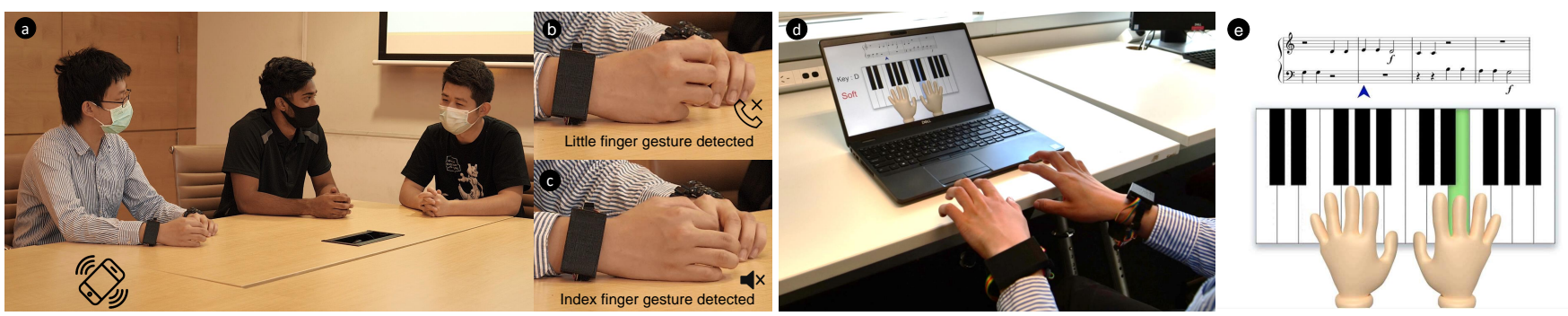

Figure 10: MyoSpring Applications - a) MyoSpring for subtle gesture interaction: A mobile phone rings during a meeting. Subtle finger gestures can be used to almost imperceptibly b) reject the call or c) mute the phone. d) Virtual Piano: Playing a virtual piano for finger rehabilitation using MyoSpring e) graphical user interface of virtual piano.

the highest accuracy for P10. Therefore, our results suggest that personalised hardware which is tailored to fit the user's specific physiological characteristics can potentially improve the system performance. While certain attributes such as BMI and wrist size have little bearing on selecting the optimal spring constant for best performance, our anecdotal observations suggest the decision should take into account more complex measurements relating to the degree of force exerted by that user when performing gestures. However, this should be further investigated to verify the impact of hardware personalisation on gesture classification accuracy.

6.1.2 Other Factors for Customisation. An important factor we believe contributes to the importance of hardware-based customisation is the different construction of individuals' wrists and hands. Numerous other factors which would have been impractical to measure, such as the strength and agility of their finger muscles, and the density or otherwise of tendons under their skin, may also affect their gesture recognition performance. These are in addition to other factors identified by research into skin viscoelasticity, including hormonal levels and past exposure to UV light. Given this degree of variance even within the tested participants, who were mostly quite close in age, the impact of hardware-level customisation would become even more pronounced within a wider population of users.

\subsection{Limitations and Future Work}

Despite the promising results of our research, there exists a number of limitations that should be addressed in future work. Primarily, future studies should aim to investigate the possibility of hardwarebased personalisation by conducting studies with users from varying backgrounds, physical characteristics and age groups to ensure generalisability of the results, and gain further insights into the key attributes that impact gesture recognition accuracy for each individual.

6.2.1 Scope for further personalisation. Our proposed MyoSpring sensors provide the potential for flexible customisation of mechanical properties to suit the needs and characteristics of individual users. In the current study, we have identified the best-performing sensor for each participant by testing three different spring types for each user and analysing the collected data. However, users may find such a procedure to be time-consuming or inconvenient. Hence, future work can conduct further advanced studies involving user characteristics, such as the physiological construction of the hand and wrist. Such studies may be able to confirm or reject our anecdotal observations on factors that can be used in determining the best-performing spring constant for a given user, in turn enabling a more streamlined and intuitive method of personalising MyoSpring sensors to each individual and increasing usability in the real world.

6.2.2 Limitations of the device. As observed in the user study, our study focused on customisation of the sensor's mechanical attributes. The current design of the MyoSpring bracelet inherently restricts the four Hall-effect sensors to be placed equidistant to each other. Due to this, it was difficult for sensors to be attached uniformly for participants with smaller wrist sizes, both in terms of the angle at which the sensors contacted the skin, as well as the initial degree of compression to which the springs were subjected. Future work should investigate this factor by allowing the distance between the sensors to be adjusted, for example by adding additional connection points between the individual sensor modules which can be achieved through a parametric 3D design tool such as Autodesk Fusion 360. This may also be aided by attaching a flexible strap over the top of the sensors so that even pressure is applied to each module. Similarly, allowing for the horizontal location of sensors along the bracelet to be adjusted for each individual user can provide further personalisation to users' mechanical structures in the carpal tunnel such as the position of specific tendons.

6.2.3 Limitations of the user study. Our study was conducted in a lab environment, which may be different from testing in real-world scenarios. While we took precautions in the data pre-processing to minimise the effects of gravity and tightness of the bracelet, further studies need to be conducted in different settings including in-thewild to further evaluate the effectiveness of MyoSpring. Our user study was conducted on fifteen users, of whom all were people without disabilities. In order to ensure generalisability and repeatability of the results, future studies should be conducted on a larger number of users, particularly to highlight whether any links between anthropometric characteristics and optimal spring stiffness would be the case among the wider population. Other studies in the past have noted the effect of additional anthropometric features such as the proportion of body fat and skinfold thickness on a wrist-worn force myographic sensor [15], thus it would also be desirable if the tested participants had a wide range of body shapes, age and other characteristics in order to investigate this further. Doing so would 
also provide more conclusive evidence for the positive wearability and comfort ratings which the participants in the current study generally provided for the device. With regard to users who have motor impairments, further studies can involve these users as participants in order to ascertain whether our solution is in fact usable for people with disabilities.

6.2.4 Limitations of the design tool. Our design tool currently only allows the user to customise the spring stiffness. While changing the spring stiffness allows for modification of the sensor characteristics, the design tool can be extended to support more customisation options. This includes the option to adjust the distance between the sensors to make it a better fit for each user's wrist.

6.2.5 Generalisability. Finally, one area that may be suggested for further work is whether our sensing solution can achieve high accuracy with user-independent classification, which involves a system performing classification without relying on training samples performed by the specific user during a calibration process [75] Therefore, if a solution can achieve user-independent classification, it will likely be of greater utility in a real-world scenario where users may not wish to calibrate the device prior to use.

\section{CONCLUSION}

In this paper, we have contributed MyoSpring, a novel technique to fabricate functionally customisable, wrist-worn, mechanomyographic finger gesture recognition sensors using 3D printed springs. We show that the sensitivity of these MMG sensors can be customised by varying the properties of 3D printed springs, and present an automated design tool with a graphical user interface for novices to quickly design and fabricate MyoSpring sensors. Through a user study, we verify MyoSpring sensors can recognise finger gestures with high accuracy, including subtle gestures with an average accuracy of $91.44 \%(\mathrm{SD}=11.76 \%)$ and all gestures with an average accuracy of $94.11 \%$ ( $\mathrm{SD}=9.73 \%$ ) across all 15 users. Our study shows that customising the physical properties of the sensor does significantly affect the sensitivity of the sensing device and can lead to improvements in gesture classification accuracy, particularly for subtle partial finger flexions. We hope MyoSpring can act as a catalyst for driving research on functionally customisable sensors, not only for gesture sensing, but also in other domains of ubiquitous computing such as sports, physical activity and health monitoring.

\section{ACKNOWLEDGMENTS}

This project was funded by the Australian Research Council Discovery Early Career Award (DECRA) - DE200100479. Dr. Withana is the recipient of a DECRA funded by the Australian Government. Additional funding for the project was generously provided by the Neurodisability Assist Trust, Australia. We are grateful to all our user study participants and anonymous reviewers for their valuable feedback.

\section{REFERENCES}

[1] Efthimios Alepis and Constantinos Patsakis. 2017. Monkey says, monkey does security and privacy on voice assistants. IEEE Access 5 (2017), 17841-17851. https://doi.org/10.1109/ACCESS.2017.2747626

[2] Ana Alves-Pinto, Varvara Turova, Tobias Blumenstein, and Renée Lampe. 2016. The case for musical instrument training in cerebral palsy for neurorehabilitation. Neural plasticity 2016 (2016). https://doi.org/10.1155/2016/1072301
[3] Brian Amento, Will Hill, and Loren Terveen. 2002. The sound of one hand: A wrist-mounted bio-acoustic fingertip gesture interface. Conference on Human Factors in Computing Systems - Proceedings (2002), 724-725.

[4] Ewout A Arkenbout, Joost C F De Winter, and Paul Breedveld. 2015. Robust hand motion tracking through data fusion of 5DT data glove and nimble VR Kinect camera measurements. Sensors 15, 12 (2015), 31644-31671. https://doi.org/10. $3390 / \mathrm{s} 151229868$

[5] Riley Booth and Peter Goldsmith. 2018. A wrist-worn piezoelectric sensor array for gesture input. Fournal of Medical and Biological Engineering 38, 2 (2018), 284-295.

[6] Andreas Bulling, Ulf Blanke, and Bernt Schiele. 2014. A tutorial on human activity recognition using body-worn inertial sensors. Comput. Surveys 46, 3 (2014), 1-33. https://doi.org/10.1145/2499621

[7] Ilaria Carpinella, Johanna Jonsdottir, and Maurizio Ferrarin. 2011. Multi-finger coordination in healthy subjects and stroke patients: a mathematical modelling approach. fournal of neuroengineering and rehabilitation 8, 1 (2011), 1-20.

[8] Girma Gebre Chassie, L E Becker, and W L Cleghorn. 1997. On the buckling of helical springs under combined compression and torsion. International fournal of Mechanical Sciences 39, 6 (1997), 697-704. https://doi.org/10.1016/S00207403(96)00070-7

[9] Hyun Ju Chong, Sung-Rae Cho, Eunju Jeong, and Soo Ji Kim. 2013. Finger exercise with keyboard playing in adults with cerebral palsy: A preliminary study. Fournal of exercise rehabilitation 9, 4 (2013), 420. https://doi.org/10.12965\%2Fjer.130050

[10] Hyun Ju Chong, Soo Jeong Han, Yong Jae Kim, Hye Young Park, and Soo Ji Kim. 2014. Relationship between output from MIDI-keyboard playing and hand function assessments on affected hand after stroke. NeuroRehabilitation 35, 4 (2014), 673-680. https://doi.org/10.3233/nre-141166

[11] Maximilian Christ, Nils Braun, Julius Neuffer, and Andreas W Kempa-Liehr. 2018. Time series feature extraction on basis of scalable hypothesis tests (tsfresh-a python package). Neurocomputing 307 (2018), 72-77.

[12] James Connolly, Joan Condell, Brendan O'Flynn, Javier Torres Sanchez, and Philip Gardiner. 2017. IMU sensor-based electronic goniometric glove for clinical finger movement analysis. IEEE Sensors fournal 18, 3 (2017), 1273-1281. https: //doi.org/10.1109/JSEN.2017.2776262

[13] Ana Grasielle Dionísio Corrêa, Irene Karaguilla Ficheman, Marilena do Nascimento, and Roseli de Deus Lopes. 2009. Computer assisted music therapy: A case study of an augmented reality musical system for children with cerebral palsy rehabilitation. In 2009 Ninth IEEE International Conference on Advanced Learning Technologies. IEEE, 218-220. https://doi.org/10.1109/ICALT.2009.111

[14] Marta Sylvia Del Rio Guerra, Jorge Martin-Gutierrez, Renata Acevedo, and Sofía Salinas. 2019. Hand gestures in virtual and augmented 3D environments for down syndrome users. Applied Sciences (Switzerland) 9, 13 (2019), 1-16. https: //doi.org/10.3390/app9132641

[15] Mona Lisa Delva, Kim Lajoie, Mahta Khoshnam, and Carlo Menon. 2020. Wristworn wearables based on force myography: on the significance of user anthropometry. BioMedical Engineering OnLine 19, 1 (2020), 1-18. https://doi.org/10. 1186/s12938-020-00789-w

[16] Artem Dementyev and Joseph A Paradiso. 2014. WristFlex: low-power gesture input with wrist-worn pressure sensors. In Proceedings of the 27th annual ACM symposium on User interface software and technology. 161-166. https://doi.org/ 10.1145/2642918.2647396

[17] Huijun Ding, Qing He, Yongjin Zhou, Guo Dan, and Song Cui. 2017. An individual finger gesture recognition system based on motion-intent analysis using mechanomyogram signal. Frontiers in neurology 8 (2017), 573.

[18] K. Dorfmüller-Ulhaas and D. Schmalstieg. 2001. Finger tracking for interaction in augmented environments. Proceedings - IEEE and ACM International Symposium on Augmented Reality, ISAR 2001 (2001), 55-64. https://doi.org/10.1109/ISAR. 2001.970515

[19] Aarthi Easwara Moorthy and Kim-Phuong L Vu. 2015. Privacy concerns for use of voice activated personal assistant in the public space. International fournal of Human-Computer Interaction 31, 4 (2015), 307-335. https://doi.org/10.1080/ 10447318.2014.986642

[20] KERSTIN B EBERHARDT, B Svensson, and U Moritz. 1988. Functional assessment of early rheumatoid arthritis. Rheumatology 27, 5 (1988), 364-371.

[21] Kristian Ehlers and Konstantin Brama. 2016. A human-robot interaction interface for mobile and stationary robots based on real-time 3D human body and handfinger pose estimation. IEEE International Conference on Emerging Technologies and Factory Automation, ETFA 2016-November (2016). https://doi.org/10.1109/ ETFA.2016.7733719

[22] Valentino Frati and Domenico Prattichizzo. 2011. Using Kinect for hand tracking and rendering in wearable haptics. 2011 IEEE World Haptics Conference, WHC 2011 (2011), 317-321. https://doi.org/10.1109/WHC.2011.5945505

[23] Eoghan Furey and Juanita Blue. 2018. She knows too much-voice command devices and privacy. In 2018 29th Irish Signals and Systems Conference (ISSC). IEEE, 1-6. https://doi.org/10.1109/ISSC.2018.8585380

[24] Roberto Gatti, Andrea Tettamanti, Simone Lambiase, Paolo Rossi, and Mauro Comola. 2015. Improving hand functional use in subjects with multiple sclerosis 
using a musical keyboard: a randomized controlled trial. Physiotherapy Research International 20, 2 (2015), 100-107. https://doi.org/10.1002/pri.1600

[25] L-C Gerhardt, Alexander Lenz, Nicholas D Spencer, Thomas Münzer, and Stephan Derler. 2009. Skin-textile friction and skin elasticity in young and aged persons. Skin Research and Technology 15, 3 (2009), 288-298. https://doi.org/10.1111/j.16000846.2009.00363.x

[26] Jun Gong, Zheer Xu, Qifan Guo, Teddy Seyed, Xiang Anthony Chen, Xiaojun $\mathrm{Bi}$, and Xing Dong Yang. 2018. WrisText: One-handed text entry on smartwatch using WrisT gestures. Conference on Human Factors in Computing Systems Proceedings 2018-April (2018), 1-14. https://doi.org/10.1145/3173574.3173755

[27] Mark A Hall. 1999. Correlation-based Feature Selection for Machine Learning April (1999).

[28] Nur Al-huda Hamdan, Adrian Wagner, Simon Voelker, Jürgen Steimle, and Jan Borchers. 2019. Springlets: Expressive, flexible and silent on-skin tactile interfaces. In Proceedings of the 2019 CHI Conference on Human Factors in Computing Systems. 1-14. https://doi.org/10.1145/3290605.3300718

[29] Liang He, Huaishu Peng, Michelle Lin, Ravikanth Konjeti, François Guimbretière, and Jon E Froehlich. 2019. Ondulé: Designing and Controlling 3D Printable Springs. In Proceedings of the 32nd Annual ACM Symposium on User Interface Software and Technology. 739-750. https://doi.org/10.1145/3332165.3347951

[30] Daniel Herren. [n.d.]. The Proximal Interphalangeal Joint: Arthritis and Deformity. 4, 6 ([n.d.]), 254-262. https://doi.org/10.1302/2058-5241.4.180042 arXiv:31312517

[31] Fang Hu, Peng He, Songlin Xu, Yin Li, and Cheng Zhang. 2020. FingerTrak: Continuous 3D hand pose tracking by deep learning hand silhouettes captured by miniature thermal cameras on wrist. Proceedings of the ACM on Interactive, Mobile, Wearable and Ubiquitous Technologies 4, 2 (2020), 1-24.

[32] Wolfgang Hürst and Casper Van Wezel. 2013. Gesture-based interaction via finger tracking for mobile augmented reality. Multimedia Tools and Applications 62, 1 (2013), 233-258. https://doi.org/10.1007/s11042-011-0983-y

[33] Alexandra Ion, Johannes Frohnhofen, Ludwig Wall, Robert Kovacs, Mirela Alistar, Jack Lindsay, Pedro Lopes, Hsiang Ting Chen, and Patrick Baudisch 2016. Metamaterial mechanisms. UIST 2016 - Proceedings of the 29th Annual Symposium on User Interface Software and Technology (2016), 529-539. https://doi.org/10.1145/2984511.2984540

[34] Yasha Iravantchi, Mayank Goel, and Chris Harrison. 2019. BeamBand: Hand gesture sensing with ultrasonic beamforming. Conference on Human Factors in Computing Systems - Proceedings (2019), 1-10. https://doi.org/10.1145/3290605. 3300245

[35] David Jack, Rares Boian, Alma S Merians, Marilyn Tremaine, Grigore C Burdea, Sergei V Adamovich, Michael Recce, and Howard Poizner. 2001. Virtual reality-enhanced stroke rehabilitation. IEEE transactions on neural systems and rehabilitation engineering 9, 3 (2001), 308-318. https://doi.org/10.1109/7333.948460

[36] Godwin Ponraj Joseph Vedhagiri, Xin Zhi Wang, Kirthika Senthil Kumar, and Hongliang Ren. 2020. Comparative Study of Machine Learning Algorithms to Classify Hand Gestures from Deployable and Breathable Kirigami-Based Electrical Impedance Bracelet. Multimodal Technologies and Interaction 4, 3 (2020), 47. https://doi.org/10.3390/mti4030047

[37] Pyeong-Gook Jung, Gukchan Lim, Seonghyok Kim, and Kyoungchul Kong. 2015 A wearable gesture recognition device for detecting muscular activities based on air-pressure sensors. IEEE Transactions on Industrial Informatics 11, 2 (2015), 485-494.

[38] Hsin-Liu (Cindy) Kao, Artem Dementyev, Joseph A. Paradiso, and Chris Schmandt. 2015. NailO: Fingernails as an Input Surface. Proceedings of the 33rd Annual ACM Conference on Human Factors in Computing Systems - CHI '15 (2015), 3015-3018. https://doi.org/10.1145/2702123.2702572

[39] Yoshihiro Kawahara, Steve Hodges, Benjamin S. Cook, Cheng Zhang, and Gregory D. Abowd. 2013. Instant inkjet circuits: Lab-based inkjet printing to support rapid prototyping of ubicomp devices. UbiComp 2013 - Proceedings of the 2013 ACM International foint Conference on Pervasive and Ubiquitous Computing (2013), 363-372. https://doi.org/10.1145/2493432.2493486

[40] David Kim, Otmar Hilliges, Shahram Izadi, Alex Butler, Jiawen Chen, Iason Oikonomidis, and Patrick Olivier. 2012. Digits: Freehand 3D interactions anywhere using a wrist-worn gloveless sensor. UIST'12 - Proceedings of the 25th Annual ACM Symposium on User Interface Software and Technology (2012), 167176. https://doi.org/10.1145/2380116.2380139

[41] Gierad Laput, Robert Xiao, and Chris Harrison. 2016. Viband: High-fidelity bioacoustic sensing using commodity smartwatch accelerometers. In Proceedings of the 29th Annual Symposium on User Interface Software and Technology. Tokyo, Japan, 321-333. https://doi.org/10.1145/2984511.2984582

[42] Xiangpeng Liang, Rami Ghannam, and Hadi Heidari. 2018. Wrist-worn gesture sensing with wearable intelligence. IEEE Sensors fournal 19, 3 (2018), 1082-1090. https://doi.org/10.1109/TII.2017.2779814

[43] Jaime Lien, Nicholas Gillian, M. Emre Karagozler, Patrick Amihood, Carsten Schwesig, Erik Olson, Hakim Raja, and Ivan Poupyrev. 2016. Soli: Ubiquitous gesture sensing with millimeter wave radar. ACM Transactions on Graphics 35, 4 (2016). https://doi.org/10.1145/2897824.2925953

[44] Meng-Kun Liu, Yu-Ting Lin, Zhao-Wei Qiu, Chao-Kuang Kuo, and Chi-Kang Wu. 2020. Hand Gesture Recognition by a MMG-based Wearable Device. IEEE Sensors fournal (2020). https://doi.org/10.1109/JSEN.2020.3011825

[45] Jess McIntosh, Asier Marzo, and Mike Fraser. 2017. Sensir: Detecting hand gestures with a wearable bracelet using infrared transmission and reflection. In Proceedings of the 30th Annual ACM Symposium on User Interface Software and Technology. 593-597. https://doi.org/10.1145/3126594.3126604

[46] Jess McIntosh, Asier Marzo, Mike Fraser, and Carol Phillips. 2017. Echoflex: Hand gesture recognition using ultrasound imaging. In Proceedings of the 2017 CHI Conference on Human Factors in Computing Systems. 1923-1934. https: //doi.org/10.1145/3025453.3025807

[47] Jess McIntosh, Charlie McNeill, Mike Fraser, Frederic Kerber, Markus Löchtefeld, and Antonio Krüger. 2016. EMPress: Practical hand gesture classification with wrist-mounted EMG and pressure sensing. In Proceedings of the 2016 CHI Conference on Human Factors in Computing Systems. 2332-2342. https: //doi.org/10.1145/2858036.2858093

[48] Dickson Neoh, Khairul Salleh Mohamed Sahari, and Wan Zul Fahmi Wan Ibrahim. 2018. A Dataglove Hardware Design and Real-Time Sign Gesture Interpretation.. In 2018 foint 10th International Conference on Soft Computing and Intelligent Systems (SCIS) and 19th International Symposium on Advanced Intelligent Systems (ISIS). IEEE, 946-949. https://doi.org/10.1109/SCIS-ISIS.2018.00157

[49] Aditya Shekhar Nittala, Anusha Withana, Narjes Pourjafarian, and Jürgen Steimle. [n.d.]. Multi-touch skin: A thin and flexible multi-touch sensor for on-skin input. In Proceedings of the 2018 CHI Conference on Human Factors in Computing Systems. 1-12.

[50] Hyunjoo Oh, Tung D. Ta, Ryo Suzuki, Mark D. Gross, Yoshihiro Kawahara, and Lining Yao. 2018. PEP (3D Printed Electronic Papercrafts): An Integrated Approach for 3D Sculpting Paper-Based Electronic Devices. In Proceedings of the 2018 CHI Conference on Human Factors in Computing Systems - CHI '18. ACM Press, New York, New York, USA, 1-12. https://doi.org/10.1145/3173574.3174015

[51] Simon Olberding, Sergio Soto Ortega, Klaus Hildebrandt, and Jürgen Steimle. 2015. Foldio: Digital Fabrication of Interactive and Shape Changing Objects With Foldable Printed Electronics. Proceedings of the 28th Annual ACM Symposium on User Interface Software \& Technology - UIST '15 (2015), 223-232. https://doi.org/ $10.1145 / 2807442.2807494$

[52] Claudio Orizio. 1993. Muscle sound: Bases for the introduction of a mechanomyographic signal in muscle studies. Fournal of Critical Reviews in Biomedical Engineering 21, 3 (1993), 201-243.

[53] Narjes Pourjafarian, Anusha Withana, , Joe Paradiso, and Jürgen Steimle. 2019. Multi-Touch Kit: A Do-It-Yourself Technique for Capacitive Multi-Touch Sensing using a Commodity Microcontroller. The 32nd Annual ACM Symposium on User Interface Software and Technology - UIST '19 (to appear) (2019).

[54] Celestine Preetham, Girish Ramakrishnan, Sujan Kumar, Anish Tamse, and Nagendra Krishnapura. 2013. Hand talk-implementation of a gesture recognizing glove. In 2013 Texas Instruments India Educators' Conference. IEEE, 328-331. https://doi.org/10.1109/TIIEC.2013.65

[55] Jun Rekimoto. 2001. Gesturewrist and gesturepad: Unobtrusive wearable interaction devices. In Proceedings Fifth International Symposium on Wearable Computers. IEEE, 21-27.

[56] David Rempel, Matt J. Camilleri, and David L. Lee. 2014. The design of hand gestures for human-computer interaction: Lessons from sign language interpreters. International fournal of Human-Computer Studies 72, 10 (2014), 728-735. https://doi.org/10.1016/j.ijhcs.2014.05.003

[57] Martin Schmitz, Mohammadreza Khalilbeigi, Matthias Balwierz, Roman Lissermann, Max Mühlhäuser, and Jürgen Steimle. 2015. Capricate: A Fabrication Pipeline to Design and 3D Print Capacitive Touch Sensors for Interactive Objects. In Proceedings of the 28th Annual ACM Symposium on User Interface Software \& Technology - UIST '15. ACM Press, New York, New York, USA, 253-258. https://doi.org/10.1145/2807442.2807503

[58] Martin Schmitz, Martin Stitz, Florian Müller, Markus Funk, and Max Mühlhäuser. 2019. ./trilaterate: A Fabrication Pipeline to Design and 3D Print Hover-, Touch-, and Force-Sensitive Objects. In Proceedings of the 2019 CHI Conference on Human Factors in Computing Systems - CHI '19. ACM Press, New York, New York, USA, 1-13. https://doi.org/10.1145/3290605.3300684

[59] Fereshteh Shahmiri and Paul H. Dietz. 2020. ShArc: A Geometric Technique for Multi-Bend/Shape Sensing. In Proceedings of the 2020 CHI Conference on Human Factors in Computing Systems. ACM, New York, NY, USA, 1-12. https: //doi.org/10.1145/3313831.3376269

[60] Peter B Shull, Shuo Jiang, Yuhui Zhu, and Xiangyang Zhu. 2019. Hand gesture recognition and finger angle estimation via wrist-worn modified barometric pressure sensing. IEEE Transactions on Neural Systems and Rehabilitation Engineering 27, 4 (2019), 724-732. https://doi.org/10.1109/TNSRE.2019.2905658

[61] Nabeel Siddiqui and Rosa H M Chan. 2017. A wearable hand gesture recognition device based on acoustic measurements at wrist. In 201739 th Annual International Conference of the IEEE Engineering in Medicine and Biology Society (EMBC). IEEE, 4443-4446. https://doi.org/10.1109/EMBC.2017.8037842

[62] Philip T Smith, R Ryan Vallance, and Eric R Marsh. 2005. Correcting capacitive displacement measurements in metrology applications with cylindrical artifacts. Precision engineering 29, 3 (2005), 324-335. https://doi.org/10.1016/j.precisioneng. 
2004.11.010

[63] Mihai T Tarata. 2003. Mechanomyography versus electromyography, in monitoring the muscular fatigue. Biomedical engineering online 2, 1 (2003), 1-10

[64] Shigeyuki Tateno, Yiwei Zhu, and Fanxing Meng. 2019. Hand Gesture Recognition System for In-car Device Control Based on Infrared Array Sensor. 2019 58th Annual Conference of the Society of Instrument and Control Engineers of Japan, SICE 2019 (2019), 701-706. https://doi.org/10.23919/SICE.2019.8859832

[65] Hoang Truong, Shuo Zhang, Ufuk Muncuk, Phuc Nguyen, Nam Bui, Anh Nguyen, Qin Lv, Kaushik Chowdhury, Thang Dinh, and Tam Vu. 2018. CapBand: Baeryfree successive capacitance sensing wristband for hand gesture recognition. SenSys 2018 - Proceedings of the 16th Conference on Embedded Networked Sensor Systems (2018), 54-67. https://doi.org/10.1145/3274783.3274854

[66] Hsin-Ruey Tsai, Min-Chieh Hsiu, Jui-Chun Hsiao, Lee-Ting Huang, Mike Chen and Yi-Ping Hung. 2016. TouchRing: subtle and always-available input using a multi-touch ring. In Proceedings of the 18th International Conference on HumanComputer Interaction with Mobile Devices and Services Adjunct. 891-898.

[67] U Chris Ugbolue, Wei-Hsiu Hsu, Robert J Goitz, and Zong-Ming Li. 2005. Tendon and nerve displacement at the wrist during finger movements. Clinical Biomechanics 20, 1 (2005), 50-56. https://doi.org/10.1016/j.clinbiomech.2004.08.006

[68] Dau-Chung Wang, Jung-Chuan Chou, Shih-Ming Wang, Po-Lun Lu, and Lan Pin Liao. 2003. Application of a Fringe Capacitive Sensor to Small-Distance Measurement. Fapanese Fournal of Applied Physics 42, Part 1, No. 9A (sep 2003), 5816-5820. https://doi.org/10.1143/jjap.42.5816

[69] Martin Weigel, Tong Lu, Gilles Bailly, Antti Oulasvirta, Carmel Majidi, and Jürgen Steimle. 2015. Iskin: flexible, stretchable and visually customizable on-body touch sensors for mobile computing. In Proceedings of the 33rd Annual ACM Conference on Human Factors in Computing Systems. 2991-3000.

[70] Hongyi Wen, Julian Ramos Rojas, and Anind K. Dey. 2016. Serendipity: Finger gesture recognition using an off-the-shelf smartwatch. Conference on Human Factors in Computing Systems - Proceedings (2016), 3847-3851. https://doi.org/10. $1145 / 2858036.2858466$

[71] Mathias Wilhelm, Daniel Krakowczyk, Frank Trollmann, and Sahin Albayrak 2015. eRing: Multiple finger gesture recognition with one ring using an electric field. ACM International Conference Proceeding Series 25-26-June-2015 (2015), 1-6. https://doi.org/10.1145/2790044.2790047

[72] Anusha Withana, Roshan Peiris, Nipuna Samarasekara, and Suranga Nanayakkara. 2015. ZSense: Enabling shallow depth gesture recognition for greater input expressivity on smart wearables. Conference on Human Factors in Computing Systems - Proceedings 2015-April (2015), 3661-3670. https //doi.org/10.1145/2702123.2702371

[73] Jacob O. Wobbrock, Meredith Ringel Morris, and Andrew D. Wilson. 2009. UserDefined Gestures for Surface Computing. In Proceedings of the SIGCHI Conference on Human Factors in Computing Systems (Boston, MA, USA) (CHI '09). Association for Computing Machinery, New York, NY, USA, 1083-1092. https://doi.org/10. 1145/1518701.1518866

[74] Richard B Woodward, Maria J Stokes, Sandra J Shefelbine, and Ravi Vaidyanathan. 2019. Segmenting mechanomyography measures of muscle activity phases using inertial data. Scientific reports 9, 1 (2019), 1-10. https://doi.org/10.1038/s41598019-41860-4

[75] Jiahui Wu, Gang Pan, Daqing Zhang, Guande Qi, and Shijian Li. 2009. Gesture recognition with a 3-d accelerometer. In International Conference on Ubiquitous Intelligence and Computing. Springer, 25-38. https://doi.org/10.1007/978-3-64202830-4_4

[76] M H Wu and W Y Hsu. 1998. Modelling the static and dynamic behavior of a conical spring by considering the coil close and damping effects. Fournal of Sound and Vibration 214, 1 (1998), 17-28. https://doi.org/10.1006/jsvi.1997.1511

[77] Junichi Yamaoka, Mustafa Doga Dogan, Katarina Bulovic, Kazuya Saito, Yoshihiro Kawahara, Yasuaki Kakehi, and Stefanie Mueller. 2019. FoldTronics: Creating 3D Objects with Integrated Electronics Using Foldable Honeycomb Structures. In Proceedings of the 2019 CHI Conference on Human Factors in Computing Systems CHI '19. ACM Press, New York, New York, USA, 1-14. https://doi.org/10.1145/ 3290605.3300858

[78] Xiaoming Yin and Ming Xie. 2007. Finger identification and hand posture recognition for human-robot interaction. Image and Vision Computing 25, 8 (2007), 1291-1300. https://doi.org/10.1016/j.imavis.2006.08.003

[79] Colleen M Zelazny. 2001. Therapeutic instrumental music playing in hand rehabilitation for older adults with osteoarthritis: Four case studies. Fournal of Music Therapy 38, 2 (2001), 97-113. https://doi.org/10.1093/jmt/38.2.97

[80] Cheng Zhang, Qiuyue Xue, Anandghan Waghmare, Ruichen Meng, Sumeet Jain, Yizeng Han, Xinyu Li, Kenneth Cunefare, Thomas Ploetz, Thad Starner, Omer Inan, and Gregory D. Abowd. 2018. FingerPing: Recognizing fine-grained hand poses using active acoustic on-body sensing. Conference on Human Factors in Computing Systems - Proceedings 2018-April (2018), 1-10. https://doi.org/10. $1145 / 3173574.3174011$

[81] Xu Zhang, Xiang Chen, Yun Li, Vuokko Lantz, Kongqiao Wang, and Jihai Yang. 2011. A framework for hand gesture recognition based on accelerometer and EMG sensors. IEEE Transactions on Systems, Man, and Cybernetics-Part A: Systems and Humans 41, 6 (2011), 1064-1076. https://doi.org/10.1109/TSMCA.2011.2116004

[82] Yang Zhang and Chris Harrison. 2015. Tomo: Wearable, low-cost electrical impedance tomography for hand gesture recognition. In Proceedings of the 28th Annual ACM Symposium on User Interface Software \& Technology. 167-173. https: //doi.org/10.1145/2807442.2807480

[83] Yuhui Zhu, Shuo Jiang, and Peter B Shull. 2018. Wrist-worn hand gesture recognition based on barometric pressure sensing. In 2018 IEEE 15th International Conference on Wearable and Implantable Body Sensor Networks (BSN). IEEE, 181184. https://doi.org/10.1109/BSN.2018.8329688 